\title{
Pharmacogenetics in medicine: barriers, critical factors and a framework for dialogue
}

\section{"There are several major barriers to the translation of pharmacogenetic research into clinical practice. These include what we term 'the evidence conundrum', which encompasses heterogeneity around the meaning of 'clinical utility', uncertainty regarding the course of action following a genetic test result, limited incorporation of pharmacogenetics into clinical practice guidelines and practical aspects of testing..."}

The clinical application of genetic information to make individualized treatment choices is increasing, as evidenced by greater pharmacogenetic testing in specialty practices (e.g., oncology, HIV/AIDS and psychiatry) and the emergence of consumer genomics companies. Despite increased awareness, pharmacogenetics has been relatively slow in becoming incorporated into mainstream medicine. In this editorial, we highlight the challenges and critical factors for success in integrating pharmacogenetics into medicine, and introduce the pharmacogenetics 'pyramid concept' as a dialectical framework for identifying common ground and areas of difference regarding the acceptance of pharmacogenetics.

There are several major barriers to the translation of pharmacogenetic research into clinical practice. These include what we term 'the evidence conundrum', which encompasses heterogeneity around the meaning of 'clinical utility', uncertainty regarding the course of action following a genetic test result, limited incorporation of pharmacogenetics into clinical practice guidelines and practical aspects of testing (assay validity, time to results and cost). Some of these factors are less likely to be significant constraints as the field matures, but others deserve structured debate and discussion in order for the field to advance.

There are clearly different points of view regarding what constitutes a prerequisite level of evidence to support the use of a genetic test to guide clinical decision making. For example, there are frequent calls for prospective, randomized, controlled outcomes trials to demonstrate the clinical utility of pharmacogenetics. However, others would argue that this is an unreasonable evidentiary standard, especially when given robust prior information regarding the genetic marker. In fact, in many cases, this evidentiary standard of outcomes as the primary end point is a higher evidentiary burden than the standard for approving drugs to be used in patients. Randomized, controlled trials (RCTs), prospective outcomes studies and comparative effectiveness studies are likely to be resource prohibitive in the testing of most pharmacogenetic hypotheses, and may be unethical in some cases (e.g., those related to drug safety). Furthermore, if a reasonable pharmacodynamic end point is chosen, the use of proximal phenotypes (e.g., international normalized ratio, blood pressure and imaging) is certainly appropriate and could suffice to optimize the benefit/risk of drugs in individual patients. Regulatory agencies consider specific pharmacogenetic questions and place high value on the mechanistic knowledge of genotype-phenotype associations. In the context of drug safety, important regulatory considerations include the impact of the drug event, the likelihood of the event in relation to genotype (i.e., penetrance) and the proportion of patients that are likely to be affected. This allows for flexibility and realistic expectations with regard to the evidence required to make regulatory decisions concerning pharmacogenetics [1].

In addition to the evidence conundrum, the absence of a generally accepted definition of clinical utility often limits the chance of pharmacogenetics being accepted. For example, from 2005 to May 2009, there were over 500 publications regarding clinical utility. Thus, there exists an ambiguous benchmark against which genetic tests are judged. It would be difficult for pharmacogenetic testing (and most other health interventions for that matter) to be deemed useful in patient care by applying these various definitions. Apparent misinterpretation of the NIH-DOE Task Force on Genetic Testing definition of clinical utility further complicates the issue [2]. This task force defined clinical utility in terms of the benefits and risks of knowing test results. However, while demonstrated clinical utility may be needed prior to widespread

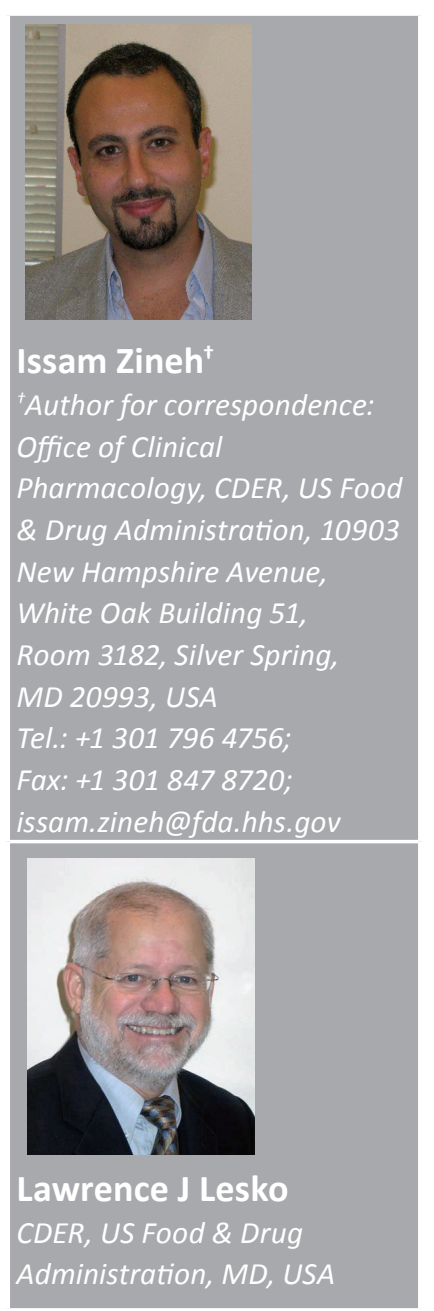

future medicue $^{\text {pass }}$ 
adoption, it is not mandated prior to use in selected patient populations in some clinical practices which may actually serve to generate additional data related to utility. Evidence regarding the clinical utility of a test should be identified by multiple stakeholders to enable efficient generation of the data needed. Furthermore, evidence of clinical utility may be demonstrated for a given practice or system (e.g., rural primary care versus tertiary hospital care) and might not necessarily be extrapolated to the healthcare system in general. One can further argue that clinical utility in the setting of personalized drug therapy choices (vis-à-vis public health interventions) is far too complex to deconvolute and includes dimensions of personal utility. To that end, the definition of clinical utility promulgated by the Secretary's Advisory Committee on Genetic Testing might better encompass genetic test usefulness [101]

'Clinical utility refers to... the value of information to the person being tested. If a test has utility, it means that the results...provide information that is of value to the person... because he or she can use that information to seek an effective treatment or preventive strategy. Even if no interventions are available to treat or prevent disease, there may be benefits associated with knowledge of a result.'

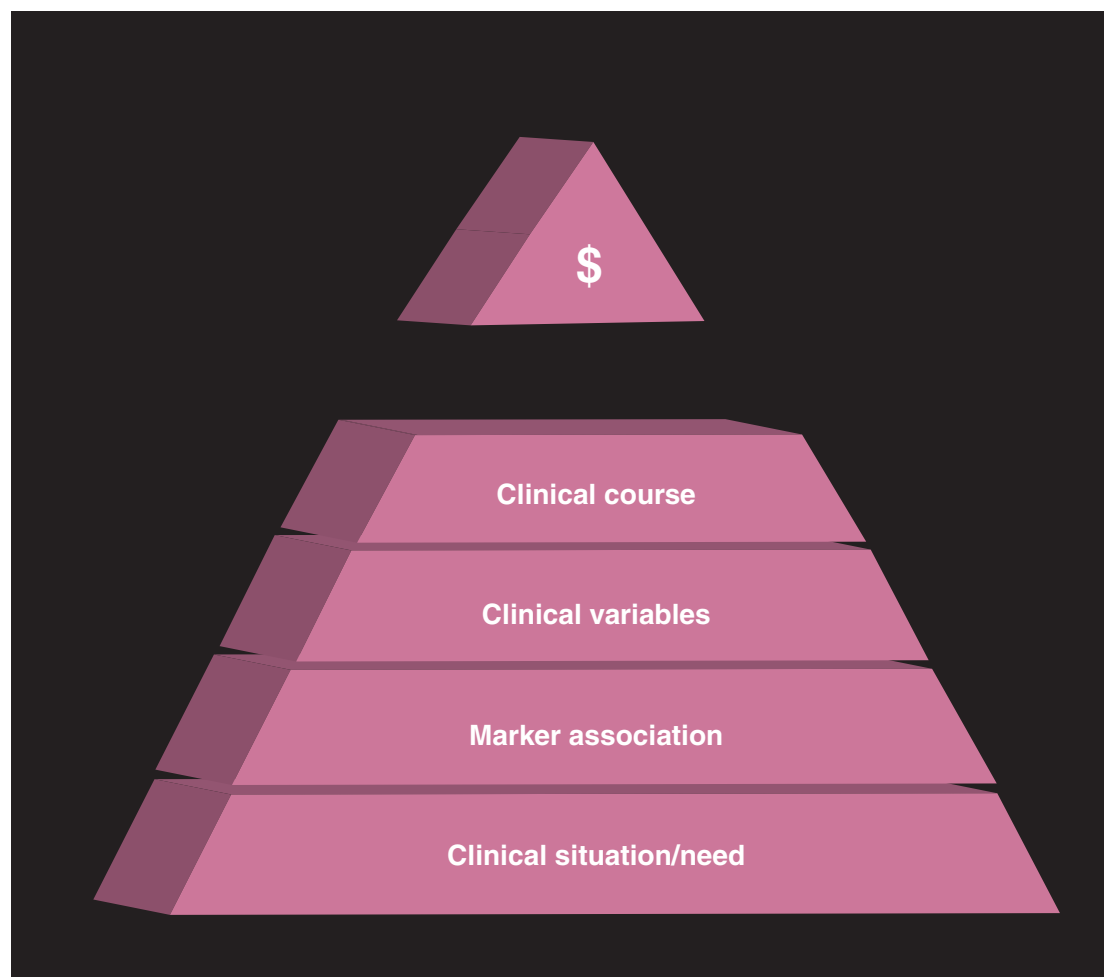

Figure 1. Pharmacogenetics pyramid. A conceptual framework for considering the value of a given genetic test in its context of use.
This definition also allows us to think more thoroughly about the use of pharmacogenetics, beyond what has been historically vague and narrowly circumscribed as medically actionable.

Despite these barriers, there have been clear examples of the adoption of genetic tests that are clinically useful. Clinicians who were hesitant to use the HIV drug abacavir, because of the potential of severe hypersensitivity reactions, more readily do so owing to a genetic test. HLA testing for variants associated with this reaction have now made it possible for clinicians to more confidently prescribe the drug. The worldwide incidence of abacavir hypersensitivity reactions has diminished and the drug has enjoyed a significant resurgence in sales [3]. In another example, the American Society of Clinical Oncology endorsed the use of $K R A S$ mutation testing in all patients with metastatic colorectal carcinoma prior to the use of anti-EGFR therapy, without a prospective RCT. Reflection on these 'success stories' reveals common themes that may more rapidly facilitate uptake into practice. These include:

- Medical specialists that are more apt to be early adopters (i.e., treaters and healers of potentially fatal diseases);

- Patients engaged in wanting to know more regarding their disease and treatments (activists);

- A technologically networked community to rapidly share knowledge;

- Incorporation of testing recommendations into clinical guidelines;

- The publication of studies in high impact journals.

RCTs, per se, are not necessarily the prerequisite to these enablers. Recognition by regulatory agencies through test approval or including test information in the drug label may also be valuable, but is not required for adoption into medical practice.

One can use an understanding of these barriers, enabling factors to construct a framework for discussing the potential value and likelihood for the successful clinical adoption of a pharmacogenetic test. Specifically, the pharmacogenetic pyramid (Figure 1) poses a series of questions whose answers incrementally inform decisions to that end. The pyramid may be applied in various situations, including clinical guideline development, label update consideration and determination by practitioners as to whether or not to use a given pharmacogenetic test. 
From the bottom up, the pyramid poses the following questions. First, what is the medical need for the genetic test? This question should take into account issues such as whether a medication is underused or underdosed owing to fear of an adverse event (e.g., abacavir hypersensitivity, major bleeding events from warfarin and severe cutaneous adverse reactions from allopurinol treatment). The severity of the adverse event, the risk of noncompliance, the absence of other ways to identify those who will benefit or those at risk and the population size of those likely to be affected may be additional considerations. Lack of efficacy (e.g., tamoxifen in CYP2D6 poor metabolizers) may be considered as a safety issue owing to the probability of a patient receiving no benefit from a drug but being exposed to adverse events. Second, what is the strength of the pharmacogenetic association? This question is meant to facilitate discussion regarding level of evidence. The robustness of findings should be rigorously determined based on factors such as the clinical and statistical strength of the association, replication of results in multiple populations, the biological gradient (i.e., ordered genotype effect) and understanding of biological plausibility. Third, what nongenetic variables are associated with the variability in response? This question aims to identify who would benefit most from testing. For example, the HLA marker associated with carbamazepine-induced Steven-Johnson syndrome is present in individuals of Asian ancestry and is almost absent in those of European ancestry. This is reflected in the current US FDA label for carbamazepine and may help target pharmacogenetic testing to appropriate populations. In the case of allopurinol hypersensitivity, elderly patients and those with decreased renal function are prone to the severe adverse event, which also has an HLA component. Therefore, HLA testing may be limited to these individuals or to those in which high doses are needed to control gout symptoms. A narrow context for the use of the genetic test rather than wholesale recommendations for testing in broad populations may be more rational and better accepted in medical practice. Fourth, what is the clinical course of action in marker-positive and/or marker-negative individuals? Depending on the issue, the availability of multiple doses of the drug of interest or alternative treatments and different monitoring recommendations are likely to address issues concerning the medical 'actionability' of a pharmacogenetic test result. Fifth, what is the cost of testing? It is important to separate this question from the rest of the pyramid since the answer is complex and not necessarily scientific. For example, regulatory agencies are required to provide information that is relevant to the improvement of public health independent of costs - the so-called benefit/risk assessment. In addition, the cost of testing and the relative cost of drugs, treatment failures and adverse events need to be accounted for in appropriately designed pharmacoeconomic studies of benefit/cost.

In summary, pharmacogenetics represents an opportunity to significantly improve the empirical way in which many patients are treated. From our perspective, barriers to pharmacogenetics uptake exist but are surmountable. Success stories help us to understand how these barriers can be overcome. A critical analysis of these factors has lead to the development of a conceptual framework for future dialogue around pharmacogenetics (FIgure 1) with the intent that focused the dialogue on 'what the questions are' and this will better facilitate the impact that pharmacogenetics can have on the day-to-day practice of clinical medicine.

\section{Disclaimer}

The views expressed are those of the authors and do not necessarily reflect the official policy of the US FDA. No official endorsement is intended or should be inferred.

\section{Financial \& competing interests disclosure}

The authors have no relevant affliations or financial involvement with any organization or entity with a financial interest in or financial conflict with the subject matter or materials discussed in the manuscript. This includes employment, consultancies, honoraria, stock ownership or options, expert testimony, grants or patents received or pending, or royalties.

No writing assistance was utilized in the production of this manuscript.

\section{Bibliography}

1 Woodcock J: The human genome and translational research: how much evidence is enough? Health Aff. (Millwood) 27, 1616-1618 (2008).

2 Holtzman NA: Promoting safe and effective genetic tests in the United States: work of the task force on genetic testing. Clin. Chem. 45, 732-738 (1999). 10, 874-878 (2008).

\section{Website}

101 Secretary's Advisory Committee on Genetics, Health and Society - Coverage and reimbursement of genetic tests and services http://oba.od.nih.gov/oba/sacghs/reports/ CR_report.pdf.

(Accessed 21 May 2009) 